\title{
The Impact of the Group Environment on the O VI Circumgalactic Medium
}

\author{
Stephanie K. Pointon ${ }^{1}$, Nikole M. Nielsen ${ }^{1}$, Glenn G. Kacprzak ${ }^{1}$, Sowgat Muzahid ${ }^{2}$, Christopher W. Churchill ${ }^{3}$, \\ and Jane C. Charlton ${ }^{4}$ \\ ${ }^{1}$ Centre for Astrophysics and Supercomputing, Swinburne University of Technology, Hawthorn, Victoria 3122, Australia; spointon@swin.edu.au \\ ${ }^{2}$ Leiden Observatory, University of Leiden, P.O. Box 9513, NL-2300 RA Leiden, The Netherlands \\ ${ }^{3}$ Department of Astronomy, New Mexico State University, Las Cruces, NM 88003, USA \\ ${ }^{4}$ Department of Astronomy and Astrophysics, The Pennsylvania State University, State College, PA 16801, USA \\ Received 2017 April 12; revised 2017 May 29; accepted 2017 June 2; published 2017 July 18
}

\begin{abstract}
We present a study comparing O VI $\lambda \lambda 1031,1037$ doublet absorption found toward group galaxy environments to that of isolated galaxies. The O VI absorption in the circumgalactic medium (CGM) of isolated galaxies has been studied previously by the "Multiphase Galaxy Halos" survey, where the kinematics and absorption properties of the CGM have been investigated. We extend these studies to group environments. We define a galaxy group as having two or more galaxies with a line-of-sight velocity difference of no more than $1000 \mathrm{~km} \mathrm{~s}^{-1}$ and located within $350 \mathrm{kpc}$ (projected) of a background quasar sightline. We identified a total of six galaxy groups associated with O VI absorption $W_{\mathrm{r}}>0.06 \AA$ that have a median redshift of $\left\langle z_{\text {gal }}\right\rangle=0.1669$ and a median impact parameter of $\langle D\rangle=134.1 \mathrm{kpc}$. An additional 12 non-absorbing groups were identified with a median redshift of $\left\langle z_{\mathrm{gal}}\right\rangle=0.2690$ and a median impact parameter of $\langle D\rangle=274.0 \mathrm{kpc}$. We find the average equivalent width to be smaller for group galaxies than for isolated galaxies $(3 \sigma)$. However, the covering fractions are consistent with both samples. We used the pixel-velocity two-point correlation function method and find that the velocity spread of O VI in the CGM of group galaxies is significantly narrower than that of isolated galaxies $(10 \sigma)$. We suggest that the warm/hot CGM does not exist as a superposition of halos; instead, the virial temperature of the halo is hot enough for $\mathrm{O}$ VI to be further ionized. The remaining O VI likely exists at the interface between hot diffuse gas and cooler regions of the CGM.
\end{abstract}

Key words: galaxies: halos - quasars: absorption lines

\section{Introduction}

The environment in which galaxies exist plays a significant part in the way they will evolve. While unperturbed isolated galaxies have a disk-like structure, interactions between galaxies can result in other interesting phenomena such as tidal streams, shells, and increased star formation rates (e.g., Barnes \& Hernquist 1992; Veilleux et al. 2005; Poggianti et al. 2016). In the most extreme cases interactions remove the galaxy gas reservoir, leading to the quenching of star formation, and the original disk-like structures of the merging galaxies deform into elliptical galaxies (e.g., Gunn \& Gott 1972; Cowie \& Songaila 1977; Larson et al. 1980; Nulsen 1982; Moore et al. 1996; Cen \& Ostriker 1999; Oppenheimer \& Davé 2008; Lilly et al. 2013). These phenomena are observed in images taken of interacting galaxies with, for example, bursts of star formation activity (e.g., Keel et al. 1985; Barnes 2004) or streams of HI gas connecting galaxies that can be seen (e.g., Bridge et al. 2010). However, we know very little about the impact of a merger or interaction on the diffuse gas around galaxies.

Of particular interest is the circumgalactic medium (CGM), which is a vast halo of diffuse gas surrounding galaxies out to radii $\approx 200$ kpc (e.g., Kacprzak et al. 2008; Chen et al. 2010; Steidel et al. 2010; Kacprzak et al. 2011; Tumlinson et al. 2011; Rudie et al. 2012; Burchett et al. 2013; Nielsen et al. 2013b, 2013a; Werk et al. 2013). The mass of this halo is comparable to the gas mass of the galaxy itself (i.e., the ISM; Thom et al. 2011; Tumlinson et al. 2011; Werk et al. 2013) and hence plays an important role in the evolution of galaxies. Models of the CGM indicate that gas can flow in and out of this reservoir, which in turn controls the star formation rate of the galaxy and the metallicity of stars formed from this gas (Oppenheimer \& Davé 2008; Lilly et al. 2013; Kacprzak et al. 2016). While a more concrete model of how this gas drives isolated galaxy evolution is being built, we are still only just beginning to study the effects that an interaction or merger can have on the CGM.

Since the visible components of galaxies are clearly affected by galaxy interactions, it is reasonable to expect that such effects would also take place in the CGM. Indeed, due to the large radius of the CGM, two galaxies could have overlapping gaseous halos even if they do not yet show any signs that an interaction is taking place (Tully et al. 2009; Bordoloi et al. 2011; Stocke et al. 2014). Thus, it is possible to use the CGM to understand the very first processes that take place in an interaction. Similarly, as interactions often result in the ISM being stripped from the galaxy leading to gas depletion (e.g., Gunn \& Gott 1972; Larson et al. 1980; Fujita \& Nagashima 1999), the CGM could also be susceptible to gas removal through tidal interactions (Chen \& Mulchaey 2009). This, in turn, would affect the ability of galaxies to form stars later in life as the accretion of gas from the CGM is what sustains star formation.

Previous studies of the CGM in group environments have used the $\operatorname{Mg}$ II $\lambda \lambda 2796,2803$ absorption doublet. Bordoloi et al. (2011) stacked background galaxy spectra and found that Mg II absorption at a given equivalent width was located at higher impact parameters in group environments compared to isolated environments. This could be explained by constructing a simple model where the CGM of the group galaxies were superimposed. Thus the extended CGM is due to more gas along the line of sight, and hence, the Mg II component of the 
CGM is minimally affected by the interactions in the group. Recent work by N. M. Nielsen et al. (2017, in preparation) has also compared $\mathrm{Mg}$ II absorption between group and isolated environments. They found that the absorption associated with group galaxies had larger equivalent widths than for isolated environments. The authors also found an increased fraction of Mg II components with higher velocities in group environments, which indicated that there could be some interaction between the CGM halos of the group galaxies.

Another ideal absorption doublet to trace the CGM is CIV, which traces gas with temperatures $T \approx 10^{4-5} \mathrm{~K}$. Burchett et al. (2016) compared C IV absorption in isolated and group/cluster environments and found that for a massive and dense group environment with seven group member galaxies $\left(M_{h}>10^{12.7} M_{\odot}\right.$ and $M_{r} \leqslant-19$ ), no $\mathrm{C}$ IV was detected despite associated $\mathrm{H} \mathrm{I}$ absorbers. They find that the cause of C IV depletion in group environments is not clear due to the limited sample size. However, if larger studies of C IV absorption follow similar trends, the authors suggest that the CGM of the group galaxies may be experiencing ram pressure or tidal stripping, and that C IV traces gas with higher temperatures than $\mathrm{HI}$ absorbers.

The warmer, more diffuse phase of the CGM gas is traced by $\mathrm{O}$ VI due to its higher ionization state and temperature $T \approx 10^{5.5} \mathrm{~K}$. O VI can be both collisionally ionized and photoionized, which makes interpretation more difficult because it traces multiphase structures (Mo \& MiraldaEscude 1996; Maller \& Bullock 2004; Dekel \& Birnboim 2006). Previous studies by Stocke et al. (2014, 2017) compared $\mathrm{O}$ VI absorption in group environments to that found in isolated environments. They found that O VI absorption profiles had to be modeled using fewer, broader components in group environments, indicating a warmer environment. However, the total absorption profile was narrower, from which the authors reasoned that $\mathrm{O}$ VI could not be distributed over the "circum-group" medium, especially since O VI is unstable due to rapid cooling. This suggests that a diffuse halo of warm CGM gas would be difficult to maintain, and that O VI exists at the interface of the diffuse hot $\left(T>10^{6} \mathrm{~K}\right)$ and cooler photoionized regions embedded in the "circum-group" medium. The existence of interfaces between hot and cold gas within the CGM has also been found by Churchill et al. (2012) and Stern et al. (2016) for isolated environments.

Recently, Oppenheimer et al. (2016) used the EAGLE simulations to compare the column density of various oxygen ionization states as a function of halo mass and found that the presence and strength of $\mathrm{O}$ VI absorption are strongly related to the mass of the halo. Halos of sub- $L^{*}$ galaxies contain little $\mathrm{O}$ VI absorption because the virial temperature is too low to ionize oxygen to such a high state. As the halo mass increases, the presence and strength of $\mathrm{O}$ VI absorption increases until the virial temperature approaches the temperature where the ionization fraction of O VI peaks, which occurs in the CGM surrounding $L^{*}$ galaxies. Finally, as the halo mass increases and virial temperature beyond this point, the presence of $\mathrm{O} \mathrm{VI}$ absorption decreases as oxygen is ionized to higher states. They find that oxygen is more likely to exist as O VII and O VIII for more massive halos, in particular halos significantly larger than $L^{*}$.

To investigate the simulation predictions from Oppenheimer et al. (2016) and compare to Mg II and CIV absorption results, we use two O VI samples, one which contains only isolated galaxies and the other which contains galaxies in group environments. The isolated sample, which represents $L^{*}$ galaxies, has been presented in the "Multiphase Galaxy Halos" survey (Kacprzak et al. 2015; Muzahid et al. 2015, 2016; Nielsen et al. 2017). Here we present the group sample, representing halos larger than that of $L^{*}$ environments, which is the combination of data from the "Multiphase Galaxy Halos" survey and the literature (Ellingson et al. 1991; Chen et al. 2001; Chen \& Mulchaey 2009; Prochaska et al. 2011; Werk et al. 2012, 2013, 2014; Johnson et al. 2013; Savage et al. 2014). A combination of HST images and HST/COS spectra allows us to investigate the equivalent widths, covering fractions, and kinematics of group environments and compare them to existing surveys of O VI absorption toward isolated galaxies.

This paper is organized as follows: In Section 2 we describe our sample of galaxies, quasars, and the selection criterion used to classify group and isolated environments. We also describe how we obtain a physical description of the CGM in such environments using quasar spectra. We present the results of our analysis with absorption properties and kinematics in Section 3. In Section 4 we discuss the differences between O VI absorption for group and isolated environments, and in Section 5 we summarize the work of this paper. We use standard a $\Lambda$ CDM cosmology $\left(H_{o}=70 \mathrm{~km} \mathrm{~s}^{-1} \mathrm{Mpc}^{-1}, \Omega_{M}=0.3\right.$, $\left.\Omega_{\Lambda}=0.7\right)$.

\section{Sample and Data}

We identified group environments in a total of 10 fields. All but three were identified in quasar spectra from the Cosmic Origins Spectrograph (COS) on HST. The remaining three were from the FUSE telescope, and the spectra were provided by B. Wakker (2016, private communication). The quasar locations, redshifts, and the observation PIDs are shown in Table 1. The quasar spectra that sample foreground galaxy groups with O VI absorption are shown in columns two and four of Figure 1 as a black line.

\subsection{Galaxy Properties}

We use a sample of galaxies located in group environments drawn from the "Multiphase Galaxy Halos" survey (Kacprzak et al. 2015; Muzahid et al. 2015, 2016; Nielsen et al. 2017) or obtained from the literature. A group is defined as having the nearest of two or more galaxies within $20^{5}$ to $350 \mathrm{kpc}$ of the quasar sightline, and the galaxies must have a line-of-sight velocity separation less than $1000 \mathrm{~km} \mathrm{~s}^{-1}$. With these criteria, we compiled a sample of six group environments with detected $\mathrm{O}$ VI absorption above a rest-frame equivalent width of $W_{r} \geqslant 0.06 \AA$ in background quasar spectra, and an additional 12 group environments with no detected absorption. Two of these group environments detected with O VI absorption were identified by Savage et al. (2014) from FUSE data. The median redshift and impact parameter of the galaxies in the group environment sample with O VI absorption are $\left\langle z_{\text {gal }}\right\rangle=0.1669$ and $\langle D\rangle=134.1 \mathrm{kpc}$, respectively. In comparison, the median values for the equivalent width and impact parameter for group environments with no $\mathrm{O}$ VI absorption are $\left\langle z_{\text {gal }}\right\rangle=0.2690$ and $\langle D\rangle=274.0 \mathrm{kpc}$. We show the group galaxy redshift, position, and impact parameter in

\footnotetext{
5 The lower limit on the impact parameter is set to match the impact parameter distribution of the isolated sample from Kacprzak et al. (2015).
} 
Table 1

Quasar Observations

\begin{tabular}{|c|c|c|c|c|c|c|}
\hline $\mathrm{J}$-Name & R.A. (J2000) & Decl. (J2000) & $z_{\mathrm{qso}}$ & Instrument & References $^{\mathrm{a}}$ & COS/FUSE PID \\
\hline $\mathrm{J} 004706+031955$ & $00: 47: 05.93$ & $+03: 19: 54.90$ & 0.6233 & $\mathrm{COS}$ & (3) & 12275 \\
\hline J012529-000556 & $01: 25: 28.84$ & $-00: 05: 55.93$ & 1.0748 & $\mathrm{COS}$ & (1) & 13398 \\
\hline J022815-405714 & 02: $28: 15.17$ & $-40: 57: 14.29$ & 0.4934 & $\cos$ & (2) & 11541 \\
\hline J035129-142909 & $03: 51: 28.54$ & $-14: 29: 08.71$ & 0.6163 & $\cos$ & (5) & 13398 \\
\hline J040748-121137 & 04: 07: 48.43 & $-12: 11: 36.66$ & 0.5726 & COS, FUSE ${ }^{\mathrm{b}}$ & (4) & 11541, B087 \\
\hline J045609-215909 & 04: 56: 08.92 & $-21:$ 59: 09.40 & 0.5335 & $\mathrm{COS}$ & (7) & 13398 \\
\hline $\mathrm{J} 092838+602521$ & 09: 28: 37.98 & $+60: 25: 21.02$ & 0.2959 & $\cos$ & (3) & 11598 \\
\hline $\mathrm{J} 111909+211918$ & 11: 19: 08.68 & $+21: 19: 18.01$ & 0.1765 & FUSE $^{\mathrm{b}}$ & (3) & P101 \\
\hline $\mathrm{J} 113328+032719$ & 11: $33: 27.78$ & $+03: 27: 19.17$ & 0.5245 & $\cos$ & (6) & 11598 \\
\hline J113910-135044 & 11: 39: 10.70 & $-13: 50: 43.64$ & 0.5565 & $\mathrm{COS}$ & (1) & 12275 \\
\hline $\mathrm{J} 130112+590206$ & 13: $01: 12.93$ & $+59: 02: 06.75$ & 0.4778 & $\cos$ & (8) & 11541 \\
\hline $\mathrm{J} 131956+272808$ & 13: 19: 56.23 & $+27: 28: 08.22$ & 1.0147 & $\cos$ & (8) & 11667 \\
\hline J135704+191907 & 13: $57: 04.43$ & $+19: 19: 07.37$ & 0.7200 & $\mathrm{COS}$ & (8) & 13398 \\
\hline $\mathrm{J} 170441+604430$ & 17: 04: 41.37 & $+60: 44: 30.50$ & 0.3719 & $\cos$ & (8) & 12276 \\
\hline $\mathrm{J} 182157+642037$ & 18: $21: 57.31$ & $+64: 20: 36.37$ & 0.2970 & $\cos$ & (8) & 12038 \\
\hline
\end{tabular}

Notes.

${ }^{a}$ Reference for the position and redshift of the quasars are: (1) Beasley et al. (2002); (2) Beuermann et al. (1999); (3) Zickgraf et al. (2003); (4) Fey et al. (2004); (5) Li \& Jin (1996); (6) Wu et al. (2012); (7) Healey et al. (2007); (8) Chen et al. (2001).

${ }^{\mathrm{b}}$ These quasar spectra were obtained from B. Wakker (2016, private communication).
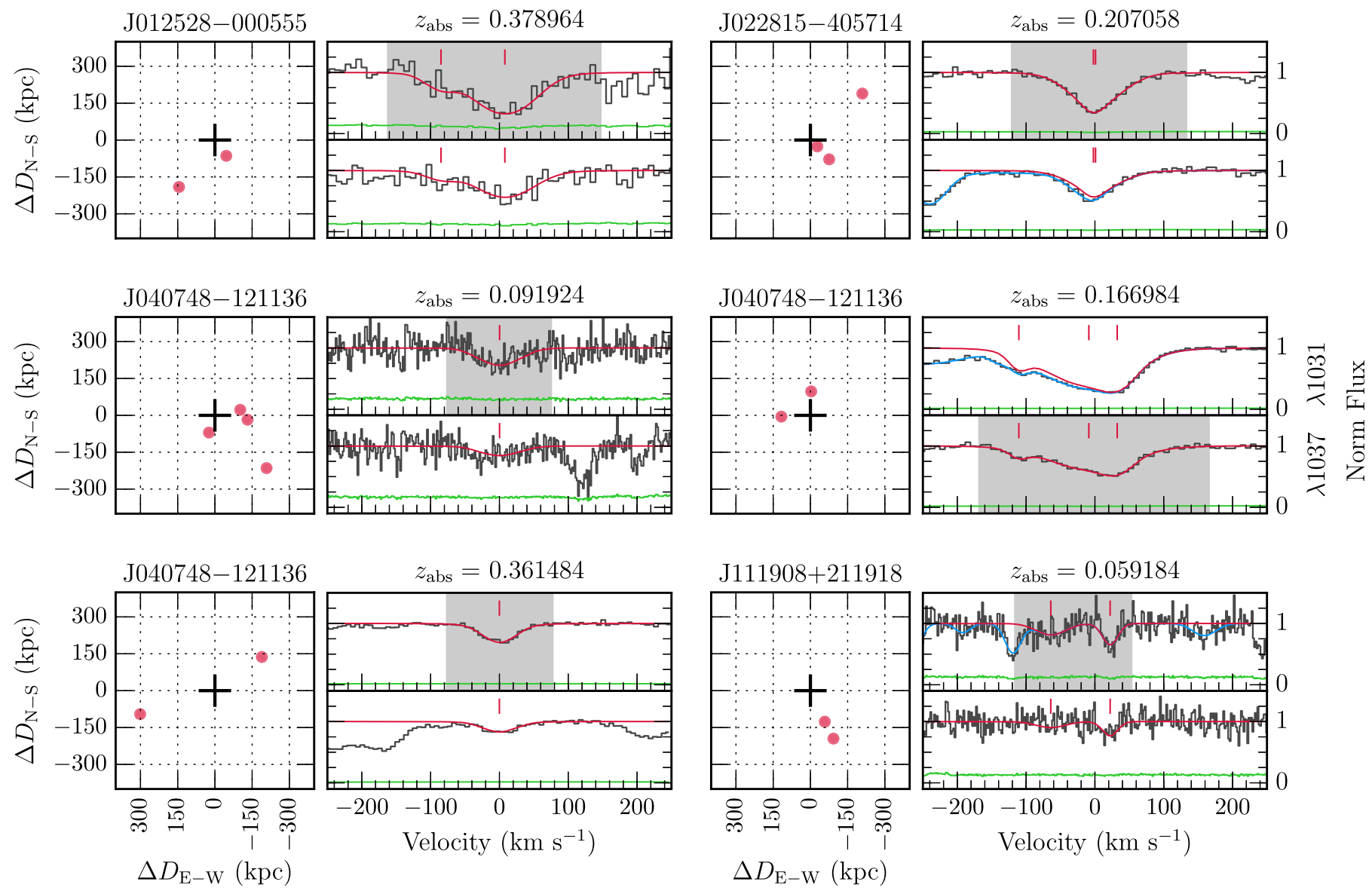

Figure 1. Sky locations and HST/COS or FUSE spectra absorption profiles for each group environment with O VI absorption. The absorbers J040748-121136 at $z_{\mathrm{abs}}=0.091917$ and $\mathrm{J} 111908+211918$ at $z_{\mathrm{abs}}=0.059188$ are from FUSE spectra. The relative positions of the galaxies in each group (red circles) are shown with respect to the locations of the quasar (black cross). The top profile shows the O VI $\lambda 1031$ absorption line, while the bottom profile shows the $\mathrm{O}$ VI $\lambda 1037$ line, where the data is shown in black, the error spectrum in green, and the O VI model in red. The center of each model component is indicated by a red tick. The blue line shows the modeled fit to the data where other transitions were modeled in addition to $\mathrm{O}$ VI due to the presence of blends. The shaded region contains the absorbing pixels within the velocity regions defined in Section 2.2. 
Table 2

Group Galaxies

\begin{tabular}{|c|c|c|c|c|c|c|c|c|c|c|c|}
\hline $\begin{array}{l}\text { (1) } \\
\text { Quasar J-name }\end{array}$ & $\begin{array}{l}\text { (2) } \\
z_{\text {gal }}\end{array}$ & $\begin{array}{c}\text { (3) } \\
\text { References }^{\mathrm{a}}\end{array}$ & $\begin{array}{l}(4) \\
\Delta \alpha \\
\left({ }^{\prime \prime}\right)\end{array}$ & $\begin{array}{l}(5) \\
\Delta \delta \\
\left({ }^{\prime \prime}\right)\end{array}$ & $\begin{array}{c}(6) \\
\theta \\
\left({ }^{\prime \prime}\right)\end{array}$ & $\begin{array}{c}(7) \\
D^{\mathrm{b}} \\
(\mathrm{kpc})\end{array}$ & $\begin{array}{l}(8) \\
z_{\text {abs }}\end{array}$ & $\begin{array}{l}(9) \\
W_{\mathrm{r}}^{\mathrm{c}} \\
(\AA)\end{array}$ & $\begin{array}{c}(10) \\
\log N(\mathrm{O} \mathrm{VI})^{\mathrm{c}}\end{array}$ & $\begin{array}{c}(11) \\
v_{-}{ }^{\mathrm{c}} \\
\left(\mathrm{km} \mathrm{s}^{-1}\right)\end{array}$ & $\begin{array}{c}(12) \\
v_{+}{ }^{\mathrm{c}} \\
\left(\mathrm{km} \mathrm{s}^{-1}\right)\end{array}$ \\
\hline \multicolumn{12}{|c|}{ Properties of Group Galaxies Associated with O VI Absorption $\left(W_{r}>0.06 \AA\right)$} \\
\hline $\mathrm{J} 012328-000555$ & $\begin{array}{l}0.3787 \\
0.3792\end{array}$ & $\begin{array}{l}5 \\
3\end{array}$ & $\begin{array}{r}-8.8 \\
27.7\end{array}$ & $\begin{array}{l}-12.3 \\
-36.5\end{array}$ & $\begin{array}{l}15.07 \\
45.80\end{array}$ & $\begin{array}{r}78.3 \\
238.1\end{array}$ & 0.378964 & $0.32 \pm 0.04$ & $14.60 \pm 0.07$ & -158 & 143 \\
\hline J022815-405714 & $\begin{array}{l}0.2065 \\
0.2078 \\
0.2077\end{array}$ & $\begin{array}{l}4 \\
4 \\
4\end{array}$ & $\begin{array}{r}-9.1 \\
-24.9 \\
-69.9\end{array}$ & $\begin{array}{r}-8.4 \\
-25.9 \\
63.2\end{array}$ & $\begin{array}{l}10.87 \\
32.04 \\
82.32\end{array}$ & $\begin{array}{r}36.8 \\
108.9 \\
279.7\end{array}$ & 0.207058 & $0.199 \pm 0.007$ & $14.38 \pm 0.01$ & -117 & 129 \\
\hline J040748-121136 & $\begin{array}{l}0.0923 \\
0.0908 \\
0.0914 \\
0.0917 \\
0.0908\end{array}$ & $\begin{array}{l}2 \\
2 \\
2 \\
2 \\
2\end{array}$ & $\begin{array}{r}13.6 \\
-61.9 \\
-78.9 \\
-123.5 \\
62.5\end{array}$ & $\begin{array}{r}-39.9 \\
13.6 \\
-10.6 \\
-127.3 \\
-252.3\end{array}$ & \begin{tabular}{r|}
42.08 \\
62.01 \\
77.89 \\
175.44 \\
259.64
\end{tabular} & $\begin{array}{r}72.3 \\
104.9 \\
132.6 \\
299.6 \\
439.4\end{array}$ & 0.091924 & $0.07 \pm 0.02$ & $13.82 \pm 0.06$ & -75 & 74 \\
\hline J040748-121136 & $\begin{array}{l}0.1670 \\
0.1669\end{array}$ & $\begin{array}{l}3 \\
3\end{array}$ & $\begin{array}{r}-1.1 \\
41.3 \\
\end{array}$ & $\begin{array}{r}34.8 \\
-1.8\end{array}$ & $\begin{array}{l}34.81 \\
40.36\end{array}$ & $\begin{array}{r}99.4 \\
115.2\end{array}$ & 0.166984 & $0.245 \pm 0.004^{\mathrm{d}}$ & $14.70 \pm 0.01^{\mathrm{d}}$ & $-170^{\mathrm{d}}$ & $156^{\mathrm{d}}$ \\
\hline J040748-121136 & $\begin{array}{l}0.3614 \\
0.3608\end{array}$ & $\begin{array}{l}2 \\
2\end{array}$ & $\begin{array}{r}-38.5 \\
61.0\end{array}$ & $\begin{array}{r}27.9 \\
-19.3\end{array}$ & $\begin{array}{l}46.87 \\
62.71\end{array}$ & $\begin{array}{l}236.4 \\
315.9\end{array}$ & 0.361484 & $0.070 \pm 0.003$ & $13.82 \pm 0.01$ & -73 & 74 \\
\hline $\mathrm{J} 111908+211918$ & $\begin{array}{l}0.0600 \\
0.0594 \\
\end{array}$ & $\begin{array}{l}1 \\
1 \\
\end{array}$ & $\begin{array}{l}-48.1 \\
-83.1 \\
\end{array}$ & $\begin{array}{l}-104.9 \\
-174.6 \\
\end{array}$ & $\begin{array}{l}117.01 \\
190.99 \\
\end{array}$ & $\begin{array}{l}135.6 \\
219.3 \\
\end{array}$ & 0.059184 & $0.06 \pm 0.02$ & $13.91 \pm 0.13$ & -115 & 52 \\
\hline \multicolumn{12}{|c|}{ Properties of Group Galaxies Associated with O VI Non-absorption $\left(W_{r}<0.06 \AA\right)^{\mathrm{e}}$} \\
\hline $\mathrm{J} 004705+031954$ & $\begin{array}{l}0.3142 \\
0.3130 \\
0.3133 \\
\end{array}$ & $\begin{array}{l}6 \\
6 \\
6 \\
\end{array}$ & $\begin{array}{r}-4.0 \\
56.0 \\
61.0 \\
\end{array}$ & $\begin{array}{l}63.0 \\
39.0 \\
71.0 \\
\end{array}$ & $\begin{array}{l}63.13 \\
68.13 \\
93.54 \\
\end{array}$ & $\begin{array}{l}290 \\
312 \\
429\end{array}$ & 0.3135 & $<0.046$ & $<13.61$ & $\cdots$ & $\cdots$ \\
\hline J004705+031954 & $\begin{array}{l}0.3810 \\
0.3786 \\
\end{array}$ & $\begin{array}{l}6 \\
6 \\
\end{array}$ & $\begin{array}{r}33.0 \\
-96.0 \\
\end{array}$ & $\begin{array}{r}41.0 \\
8.0 \\
\end{array}$ & $\begin{array}{l}52.60 \\
96.17 \\
\end{array}$ & $\begin{array}{l}274 \\
499 \\
\end{array}$ & 0.3798 & $<0.054$ & $<13.68$ & $\ldots$ & $\ldots$ \\
\hline J022815-405714 & $\begin{array}{l}0.1992 \\
0.1997 \\
0.2006 \\
\end{array}$ & $\begin{array}{l}4 \\
4 \\
4 \\
\end{array}$ & $\begin{array}{r}84.0 \\
-110.6 \\
133.7 \\
\end{array}$ & $\begin{array}{r}24.6 \\
-32.4 \\
78.4 \\
\end{array}$ & $\begin{array}{r}68.04 \\
89.60 \\
127.88 \\
\end{array}$ & $\begin{array}{l}220 \\
296 \\
419 \\
\end{array}$ & 0.1998 & $<0.011$ & $<12.94$ & $\cdots$ & $\cdots$ \\
\hline J022815-405714 & $\begin{array}{l}0.2678 \\
0.2690 \\
0.2680 \\
0.2654 \\
0.2664 \\
0.2706 \\
0.2662 \\
0.2683\end{array}$ & $\begin{array}{l}4 \\
4 \\
4 \\
4 \\
4 \\
4 \\
4 \\
4\end{array}$ & $\begin{array}{r}16.9 \\
8.5 \\
36.2 \\
93.4 \\
105.7 \\
108.4 \\
116.0 \\
61.1\end{array}$ & $\begin{array}{r}-13.0 \\
-36.7 \\
-29.2 \\
-8.5 \\
9.4 \\
15.4 \\
16.9 \\
-72.9\end{array}$ & $\begin{array}{l}18.21 \\
37.25 \\
39.98 \\
71.03 \\
80.36 \\
83.28 \\
89.24 \\
86.25\end{array}$ & $\begin{array}{r}70 \\
150 \\
160 \\
284 \\
323 \\
339 \\
345 \\
349\end{array}$ & 0.2677 & $<0.016$ & $<13.11$ & $\cdots$ & $\ldots$ \\
\hline J035128-142908 & $\begin{array}{l}0.3236 \\
0.3244 \\
0.3273 \\
\end{array}$ & $\begin{array}{l}3 \\
3 \\
3 \\
\end{array}$ & $\begin{array}{r}13.0 \\
-29.9 \\
-59.0 \\
\end{array}$ & $\begin{array}{r}-23.5 \\
18.5 \\
19.5 \\
\end{array}$ & $\begin{array}{l}26.72 \\
34.33 \\
60.31\end{array}$ & $\begin{array}{l}127 \\
164 \\
288\end{array}$ & 0.3251 & $<0.032$ & $<13.41$ & $\cdots$ & $\cdots$ \\
\hline J040748-121136 & $\begin{array}{l}0.3506 \\
0.3521 \\
0.3517\end{array}$ & $\begin{array}{l}2 \\
2 \\
2\end{array}$ & $\begin{array}{r}-26.0 \\
37.0 \\
80.5\end{array}$ & $\begin{array}{r}-40.3 \\
55.7 \\
-141.3\end{array}$ & $\begin{array}{r}47.66 \\
66.40 \\
161.78 \\
\end{array}$ & $\begin{array}{l}235 \\
329 \\
802\end{array}$ & 0.3515 & $<0.004$ & $<12.51$ & $\cdots$ & $\cdots$ \\
\hline J045608-215909 & $\begin{array}{l}0.4838 \\
0.4836 \\
0.4837 \\
\end{array}$ & $\begin{array}{l}3 \\
3 \\
3 \\
\end{array}$ & $\begin{array}{r}-1.1 \\
-20.0 \\
40.1 \\
\end{array}$ & $\begin{array}{l}-18.0 \\
-16.6 \\
-36.8 \\
\end{array}$ & $\begin{array}{l}18.03 \\
24.88 \\
52.29 \\
\end{array}$ & $\begin{array}{l}108 \\
284 \\
315 \\
\end{array}$ & 0.4837 & $<0.032$ & $<13.73$ & $\cdots$ & $\cdots$ \\
\hline J085334+434902 & $\begin{array}{l}0.0915 \\
0.0903 \\
\end{array}$ & $\begin{array}{l}4 \\
7 \\
\end{array}$ & $\begin{array}{r}-1.8 \\
14.1 \\
\end{array}$ & $\begin{array}{r}40.8 \\
-34.0 \\
\end{array}$ & $\begin{array}{l}40.81 \\
35.45 \\
\end{array}$ & $\begin{array}{l}53 \\
79 \\
\end{array}$ & 0.0909 & $<0.054$ & $<13.68$ & $\cdots$ & $\cdots$ \\
\hline J092554+400414 & $\begin{array}{l}0.2475 \\
0.2467 \\
\end{array}$ & $\begin{array}{l}8 \\
8 \\
\end{array}$ & $\begin{array}{l}-8.0 \\
-7.2\end{array}$ & $\begin{array}{l}-20.8 \\
-24.1\end{array}$ & $\begin{array}{l}21.64 \\
24.69\end{array}$ & $\begin{array}{l}81 \\
92\end{array}$ & 0.2471 & $<0.085$ & $<13.91$ & $\cdots$ & $\cdots$ \\
\hline $\mathrm{J} 092837+602521$ & $\begin{array}{l}0.1537 \\
0.1542 \\
0.1540\end{array}$ & $\begin{array}{l}8 \\
8 \\
8\end{array}$ & $\begin{array}{r}-3.5 \\
30.2 \\
67.2\end{array}$ & $\begin{array}{l}-14.7 \\
-12.1 \\
-12.3\end{array}$ & $\begin{array}{l}14.82 \\
19.19 \\
35.38\end{array}$ & $\begin{array}{l}38 \\
48 \\
89\end{array}$ & 0.1540 & $<0.111$ & $<14.06$ & $\cdots$ & $\cdots$ \\
\hline
\end{tabular}


Table 2

(Continued)

\begin{tabular}{|c|c|c|c|c|c|c|c|c|c|c|c|}
\hline $\begin{array}{l}\text { (1) } \\
\text { Quasar J-name }\end{array}$ & $\begin{array}{l}\text { (2) } \\
z_{\text {gal }}\end{array}$ & $\begin{array}{c}(3) \\
\text { References }^{\mathrm{a}}\end{array}$ & $\begin{array}{l}(4) \\
\Delta \alpha \\
(")\end{array}$ & $\begin{array}{l}(5) \\
\Delta \delta \\
\left({ }^{\prime \prime}\right)\end{array}$ & $\begin{array}{c}(6) \\
\theta \\
(")\end{array}$ & $\begin{array}{c}(7) \\
D^{\mathrm{b}} \\
(\mathrm{kpc})\end{array}$ & $\begin{array}{l}(8) \\
z_{\text {abs }}\end{array}$ & $\begin{array}{l}(9) \\
W_{\mathrm{r}}^{\mathrm{c}} \\
(\AA)\end{array}$ & $\begin{array}{c}(10) \\
\log N(\mathrm{O} \mathrm{VI})^{\mathrm{c}}\end{array}$ & $\begin{array}{c}(11) \\
v_{-}{ }^{\mathrm{c}} \\
\left(\mathrm{km} \mathrm{s}^{-1}\right)\end{array}$ & $\begin{array}{c}(12) \\
v_{+}{ }^{\mathrm{c}} \\
\left(\mathrm{km} \mathrm{s}^{-1}\right)\end{array}$ \\
\hline $\mathrm{J} 113327+032719$ & $\begin{array}{l}0.2367 \\
0.2364\end{array}$ & $\begin{array}{l}8 \\
8\end{array}$ & $\begin{array}{r}4.5 \\
-4.1\end{array}$ & $\begin{array}{l}-1.7 \\
-9.6\end{array}$ & $\begin{array}{r}4.79 \\
10.39\end{array}$ & $\begin{array}{l}18 \\
35\end{array}$ & 0.2365 & $<0.067$ & $<13.79$ & $\cdots$ & $\cdots$ \\
\hline $\mathrm{J} 113910-135043$ & $\begin{array}{l}0.3598 \\
0.3604 \\
0.3595\end{array}$ & $\begin{array}{l}3 \\
3 \\
3\end{array}$ & $\begin{array}{l}-52.4 \\
-28.0 \\
-45.9\end{array}$ & $\begin{array}{l}13.3 \\
51.7 \\
38.5\end{array}$ & $\begin{array}{l}52.57 \\
58.41 \\
58.95\end{array}$ & $\begin{array}{l}265 \\
296 \\
298\end{array}$ & 0.3599 & $<0.027$ & $<13.36$ & $\cdots$ & $\cdots$ \\
\hline
\end{tabular}

Notes.

${ }^{\mathrm{a}}$ Galaxy identification reference: (1) Prochaska et al. (2011); (2) Johnson et al. (2013); (3) Chen et al. (2001); (4) Chen \& Mulchaey (2009); (5) Muzahid et al. (2015); (6) Ellingson et al. (1991); (7) Lanzetta et al. (1995); (8) Werk et al. (2012).

${ }^{\text {b }}$ We include known galaxies with $D>350 \mathrm{kpc}$ for completeness.

${ }^{\mathrm{c}}$ Unless otherwise noted, values were calculated from the $\mathrm{O}$ VI $\lambda 1031$ line.

${ }^{\mathrm{d}}$ Values were calculated from the O VI $\lambda 1037$ line.

${ }^{\mathrm{e}}$ We quote the $3 \sigma$ limits for $W_{r}$ and $\log N(\mathrm{O} \mathrm{VI})$.

Table 2 as well as the same values for the non-absorbing groups. The values for the right ascension and declination offsets and the angular separation of the galaxies from the quasar sightline, as well at the impact parameter, were recalculated using methods from Nielsen et al. (2013b). In Figure 1 we show the locations of the group galaxy members associated with OVI absorption (red points) with relation to the quasar sightline (central black cross) in the first and third columns. Similarly, we show in Figure 2 the locations of galaxies that were not associated with O VI absorption (red points) relative to the location of the quasar sightline (central black cross).

We compare this group environment sample to the isolated galaxy sample presented in Kacprzak et al. (2015), who identified 53 isolated galaxies associated with 29 absorbers and 24 non-absorbers. However, the Kacprzak et al. (2015) sample only extends out to $200 \mathrm{kpc}$. We supplement this sample with an additional 14 isolated galaxies from the "Multiphase Galaxy Halos" survey to extend the impact parameter range out to $350 \mathrm{kpc}$. These additional galaxies are presented in Table 3. The galaxies in this sample have a median redshift and impact parameter of $\left\langle z_{\text {gal }}\right\rangle=0.2608$ and $\langle D\rangle=259 \mathrm{kpc}$, respectively. We find that the galaxies in this extended impact parameter range are all identified as $\mathrm{O}$ VI non-absorbers.

\subsection{Modeling}

We performed a Voigt profile analysis using the software VPFIT $^{6}$ (Carswell \& Webb 2014) to fit the absorption profiles of the O VI absorbers. As the COS instrument on the HST has a non-Gaussian line spread function (LSF), leading to significant wings that are prominent at short wavelengths, we use the nonGaussian LSF from Kriss (2011). The LSF for each absorption profile was calculated by interpolating the LSF from Kriss (2011) to the central wavelength of the profile. This LSF was then convolved with the model profile in the fitting process. The velocity resolution of the FUSE observations is $\sim 15-20 \mathrm{~km} \mathrm{~s}^{-1}$ (FWHM) and we assume a Gaussian LSF.

Where possible, both lines of the O VI $\lambda \lambda 1031,1037$ doublet were used to fit the absorption profile to better constrain the number of components required and determine any blends that might be present. The number of components was based on the

\footnotetext{
6 https://www.ast.cam.ac.uk/ rfc/vpfit.html
}

minimum number required to produce a reasonably minimized chi-squared value. We present the results of the fitting in the second and fourth columns of Figure 1. The black line represents the data, while the red line is the model of the O VI absorption profile made using VPFIT. Red ticks indicate the central position of each component used to fit the O VI absorption profile. Three absorbers were found to have blends present. In these cases, we used the unblended transition to fit the profile. The blended transition was then modeled by adding generic components. The total fit in these two instances is shown as a blue line.

From the fitting process, we extracted the column density, Doppler $b$ parameter, and redshift of each component of the absorption profiles. We then calculated the equivalent width by integrating the absorption features between the points where the model deviated from the continuum, which we defined as the edges of the absorption profile. These were converted into velocity boundaries using $z_{\mathrm{abs}}$, which is the median velocity of the apparent optical depth distribution of the absorption and defines the velocity zero point (Churchill 1997). The regions contained by these velocity boundaries are shown as the shaded regions in Figure 1. We list the equivalent width, column density, $z_{\mathrm{abs}}$, and the absorption velocity range in Table 2 .

We also calculated $3 \sigma$ upper limits on the equivalent widths and column densities of the non-absorbing galaxies and group environments. To compute the column density limits, we assumed a single cloud with a Doppler $b$ parameter of $30 \mathrm{~km} \mathrm{~s}^{-1}$, which we found to be typical of O VI absorption in our sample. The equivalent width and column density limits for group environments and isolated galaxy are shown in Tables 2 and 3, respectively.

\section{Results}

Here we present an analysis of the equivalent width, covering fractions and kinematics of both group and isolated galaxies to determine whether environment plays a role in shaping the properties of the CGM.

\subsection{Equivalent Width and Covering Fraction}

Kacprzak et al. (2015) found that the equivalent width decreases as the distance of the absorber from the galaxy 

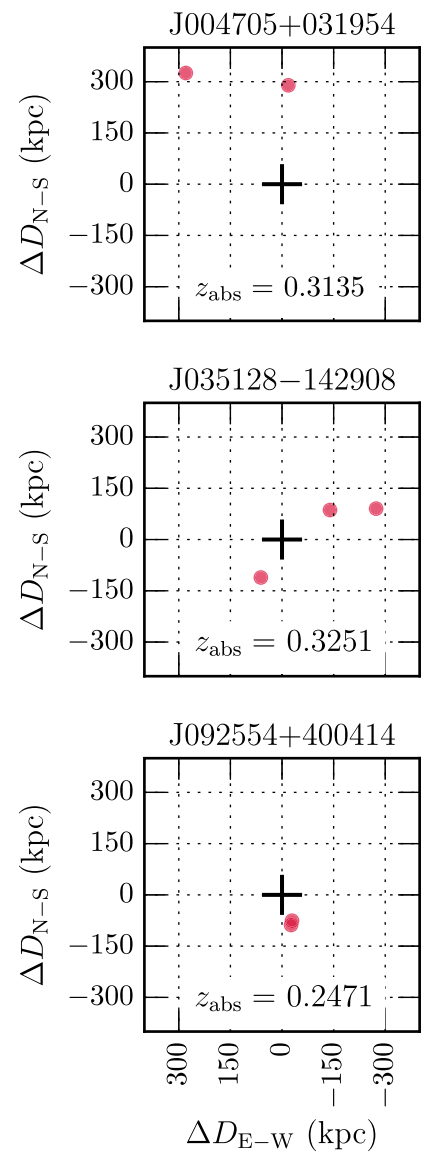
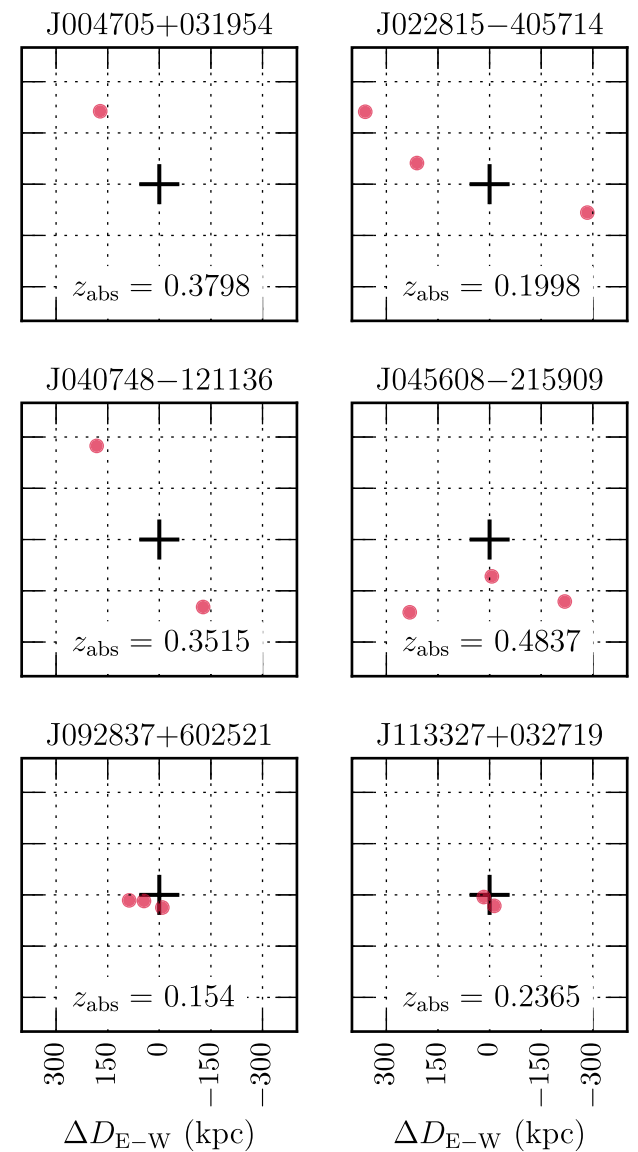
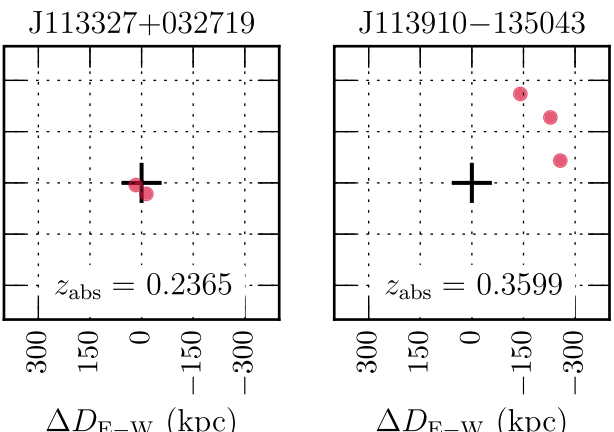

$\Delta D_{\mathrm{E}-\mathrm{W}}(\mathrm{kpc})$

Figure 2. Sky locations for each group environment with no associated O VI absorption $W_{r}<0.06 \AA$. The relative positions of the galaxies in each group (red circles) are shown with respect to the locations of the quasar (black cross).

increases for the isolated sample. Similarly, Tumlinson et al. (2011) and Johnson et al. (2015) found that the column density decreases as the ratio of the impact parameter and the virial radius increases. We investigate this trend in group galaxies by showing the equivalent width as a function of the impact parameter in Figure 3. In group environments, the impact parameter is taken to be the projected distance from the quasar to the nearest galaxy. The nearest group members are shown in orange, with circles indicating measurements of the equivalent width and crosses representing limits. In our sample, we detect absorbers with equivalent widths greater than $0.06 \AA$, which we choose to be the cut-off between absorbers and non-absorbers, represented as a black dashed line. The nearest group galaxies also appear to exhibit decreasing equivalent widths with increasing impact parameter, similar to the trend found in isolated environments. We also investigate the effect of measuring the impact parameter to the geometric center and the most massive (luminous) galaxy and find that the variations did not change the relationship significantly. Figure 3 suggests that the equivalent width of group galaxies is lower than what is expected from isolated galaxies.

We compare the equivalent width distributions of the group and isolated galaxies in Figure 4. The peak of the equivalent width distribution of group environments appears to be shifted lower than the peak for isolated galaxies. The average equivalent width for group environments is $\left\langle W_{r}\right\rangle=0.16 \pm 0.02 \AA$, while the average equivalent width for isolated galaxies is $\left\langle W_{r}\right\rangle=0.31 \pm 0.03 \AA$. This is a $3 \sigma$ difference in the mean equivalent widths. We also test the removal of the outlier from the isolated galaxy sample at the top of the plot in Figure 3 due to its unusually large equivalent width (Muzahid et al. 2015). The average equivalent width for isolated galaxies then is $\left\langle W_{r}\right\rangle=0.28 \pm 0.02 \AA$. The difference between the means of the equivalent widths of the group and isolated environments is then $2.4 \sigma$.

The covering fraction, which is the ratio of absorbers to the total number of systems, is an indication of a lack of gas or ideal ionizing conditions in the CGM. We find that the covering fraction for group galaxies is $C_{f}=0.33_{-0.11}^{+0.06}$, while the isolated galaxies have $C_{f}=0.43_{-0.06}^{+0.03}$, where the $1 \sigma$ errors are derived from 10,000 bootstrap realizations. These are consistent within the errors of the measurements $(0.8 \sigma)$, although the smaller covering fraction for the group galaxies suggests that the CGM in these environments appears to have a lack of gas or insufficient ionizing conditions compared to isolated environments. Lower equivalent widths and covering fractions for group galaxies could indicate that these environments are being depleted of $\mathrm{O}$ VI and a larger sample would be invaluable to investigate this effect.

\subsection{Pixel-velocity Two-point Correlation Function}

To compare the kinematics of the $\mathrm{O}$ VI absorbers for group and isolated environments, we use the pixel-velocity two-point correlation function (TPCF) method from Nielsen et al. (2015, 2016, 2017). To construct the isolated galaxy TPCF, Nielsen et al. (2017) compiled the pixel velocities within the absorption velocity bounds (i.e., shaded regions in Figure 1) for every isolated absorber in the sample. With this list, they calculated 
Table 3

Properties of Isolated Galaxies with $200 \mathrm{kpc} \leqslant D \leqslant 350 \mathrm{kpc}$

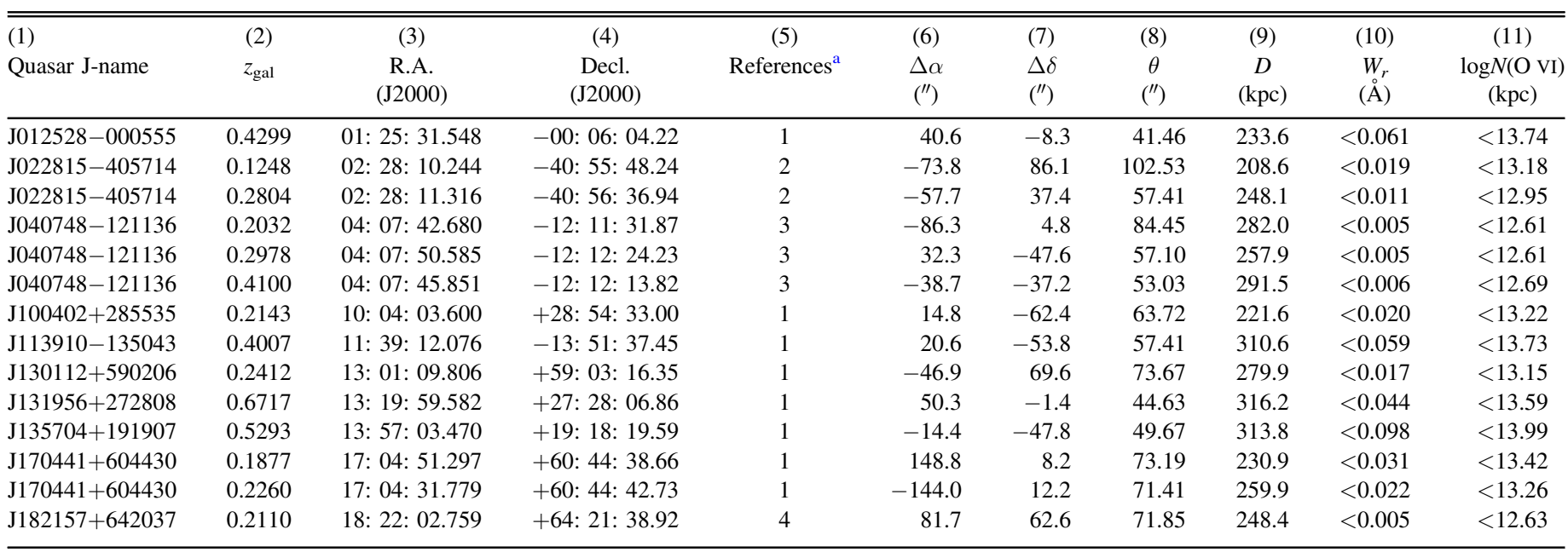

Note.

${ }^{a}$ Galaxy identification reference: (1) Chen et al. (2001); (2) Chen \& Mulchaey (2009); (3) Johnson et al. (2013); (4) Stocke et al. (2013).

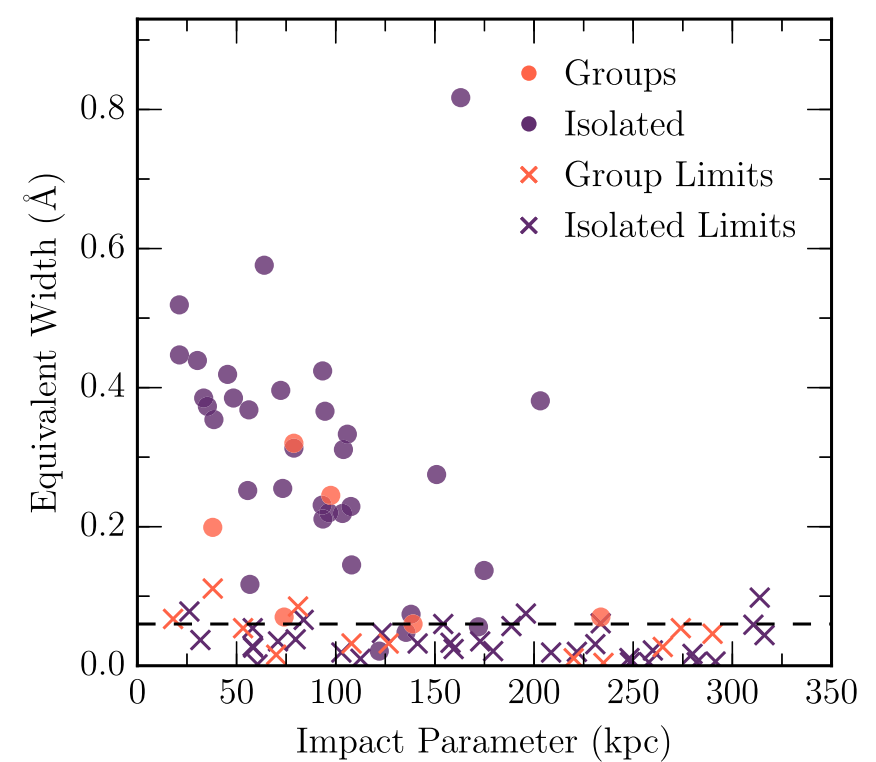

Figure 3. Equivalent widths of each $\mathrm{O}$ VI $\lambda 1031$ profile as a function of impact parameter to the nearest member galaxy. The nearest group galaxies are shown in orange and isolated galaxies in purple. Circles indicate where measurements of the equivalent width were possible, while crosses show where only a limit could be obtained. The black dashed line indicates the cut-off between absorbers and non-absorbers $(0.06 \AA)$.

the absolute value of the velocity separations between every pixel pair, binned these velocity separations into $20 \mathrm{~km} \mathrm{~s}^{-1}$ bins, and normalized the distribution by the total number of pixel pairs in the sample. This produced a probability distribution function, which describes the probability that any two pixels will have a given velocity separation. They used a bootstrap analysis with 100 realizations to obtain $1 \sigma$ uncertainties on the TPCF. We use the same method to calculate the group TPCF.

The group galaxy TPCF (orange) and the Nielsen et al. (2017) isolated galaxy TPCF (purple) are shown in Figure 5, where the TPCF is the thick solid line and the shaded regions represent the one sigma errors calculated from the bootstrap analysis. Any two

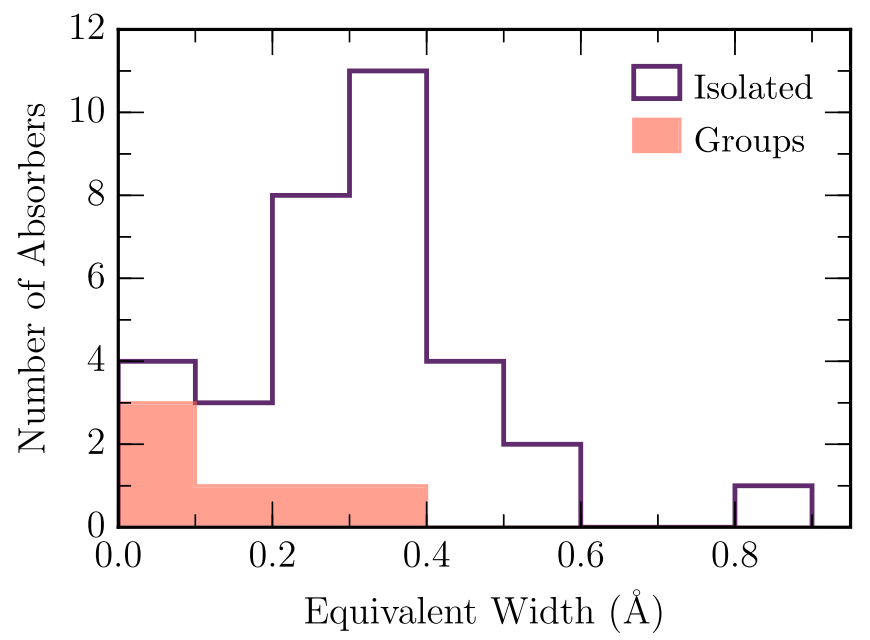

Figure 4. Equivalent width distribution of group absorbers (orange histogram) compared to the isolated absorbers (purple histogram). The group absorbers may be offset from the main peak of the isolated absorbers.

pixels for a typical group absorption environment are more likely to be closer together in velocity space than two randomly selected pixels from an isolated galaxy. A chi-squared test run on the binned TPCFs, including the uncertainties, found that the null hypothesis that the two samples were drawn from the same population can be ruled out at the $10 \sigma$ level. Thus, the O VI absorption velocity dispersion is smaller in group environments than in isolated environments.

To characterize the differences in the TPCFs quantitatively, we calculate $\Delta v(90)$ and $\Delta v(50)$ values, which are defined as the velocity separations within which the integrated TPCF curve contains $90 \%$ and $50 \%$ of the data, respectively. Thus, $\Delta v(90)$ and $\Delta v(50)$ are also measures of the velocity spreads of the distributions. We find that $\Delta v(90)$ is $153_{-18}^{+21} \mathrm{~km} \mathrm{~s}^{-1}$ for group galaxy absorption, compared to $236 \pm 15 \mathrm{~km} \mathrm{~s}^{-1}$ for isolated galaxy absorption $(2.3 \sigma)$. Similarly, the group absorption $\Delta v(50)$ is $64_{-7}^{+9} \mathrm{~km} \mathrm{~s}^{-1}$, while the isolated galaxy absorption is $100 \pm$ $6 \mathrm{~km} \mathrm{~s}^{-1}(2.4 \sigma)$. Even though the uncertainties on these values 


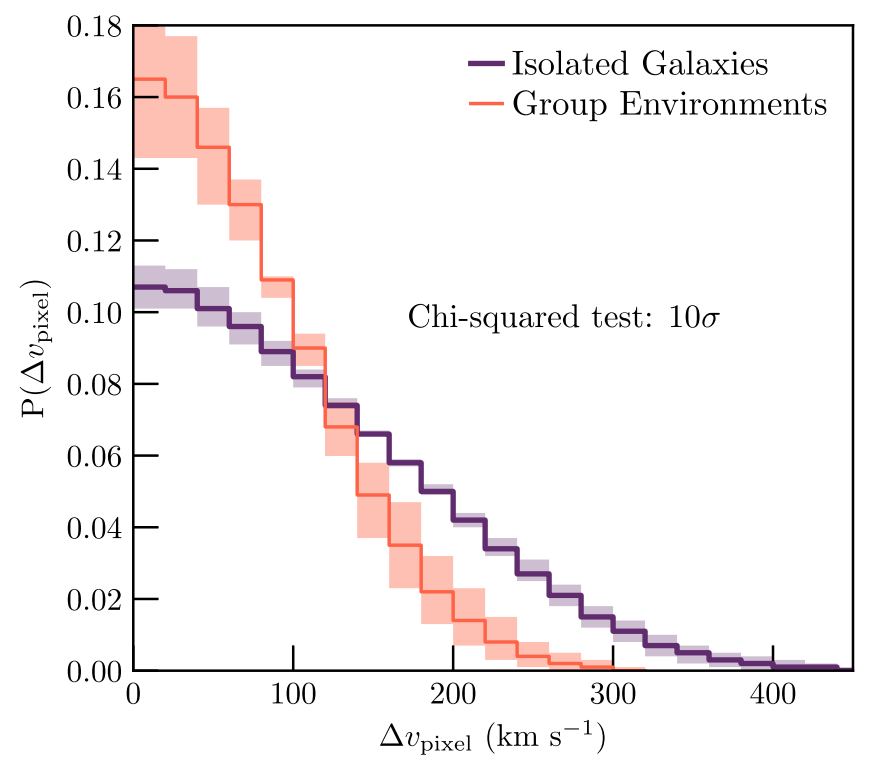

Figure 5. Pixel-velocity two-point correlation function (TPCF) comparing the velocity spread of the O VI $\lambda 1031$ absorption profiles for the group and isolated environments. The group galaxy TPCF is shown in orange, while the isolated galaxy TPCF is shown in purple. The result of the chi-squared test is $10 \sigma$, indicating that the $\mathrm{O}$ VI velocity spread of group absorption profiles is significantly narrower than the isolated TPCF. The shaded region shows the $1 \sigma$ errors calculated from bootstrapping.

do not overlap, they are still only $2.4 \sigma$ different. Thus, combined with the chi-squared test of the TPCF profiles, which is more sensitive to velocity differences in the absorption profiles (Nielsen et al. 2015, 2016, 2017), we find that group environments are more likely to have narrower absorption line profiles than isolated environments.

\section{Discussion}

The UV spectra from $H S T /$ COS and FUSE have allowed us to compare the OVI CGM in the environment surrounding groups of galaxies to the CGM of isolated galaxies. We have investigated the effects of group environments on the equivalent widths, the covering fractions, and the velocity spread of the O VI absorption profiles.

Differences between the CGM in group and isolated environments have been explored using $\mathrm{Mg}$ II. Bordoloi et al. (2011) found that for a given equivalent width, the absorption associated with group galaxies exists out to larger impact parameters than for isolated galaxies. This is supported by Chen et al. (2010), who did not find a strong inverse correlation between equivalent width and impact parameter. Bordoloi et al. (2011) suggested that this is a result of the CGM of group members being superimposed with little interaction between the halos. N. M. Nielsen (2017, in preparation) found that the velocity spread of absorption profiles for group environments is larger than the velocity spread for isolated galaxies. They also found that a higher fraction of high-velocity components (relative to $z_{\text {abs }}$ ) exist in group halos. This could suggest some interaction occurring between the halos of the group galaxies. We study O VI absorption to investigate the influence of group environments on the warm/hot CGM.

Using the EAGLE simulations, Oppenheimer et al. (2016) found interesting results with mass that may explain the difference between O VI absorption from the CGM surrounding group and isolated environments. By examining simulated $\mathrm{O}$ VI column densities, the authors found that halo mass, and therefore virial temperature, governs the presence and strength of O VI, where sub- $L^{*}$ halos do not have sufficient virial temperatures to create significant amounts of O VI. The most favorable halo masses, and hence virial temperatures, for the creation of O VI are from $L^{*}$ galaxies, which are represented by our isolated sample. As the halo mass increases above that of a typical $L^{*}$ galaxy to group and cluster environments, the amount of O VI absorption decreases due to the temperatures in the halo preferentially ionizing O VI to higher states, such as $\mathrm{O}$ VII, which are difficult to observe. In the case of studying group galaxies, the halo mass is certainly larger that this limit, hence we should start to see this effect.

The effects of group environments on absorption properties such as covering fractions and equivalent widths were investigated to determine if the trends suggested by Oppenheimer et al. (2016) were visible in our sample. Taking these results into account, we expected that the equivalent widths and covering fractions for our group sample would be lower than for isolated sample if the virial temperatures were higher in the group sample. The average equivalent width of the group sample is lower than the isolated galaxies $(3 \sigma)$. However, the covering fractions of the absorption associated with group environments, while lower than what were found for isolated galaxies, are consistent with the values from Kacprzak et al. $(2015 ; 0.8 \sigma)$. This suggests that the mass trends with the presence of O VI could be present but are limited by the small number of group systems in this study. The unchanged covering fraction for group samples may be due to interactions between the group members stripping gas from the galaxies into the CGM. Further investigations should focus on improving the sample size of group environments to determine if groups environments have significantly smaller covering fractions.

The effects of group environments on C IV was investigated by Burchett et al. (2016), who found that groups with more galaxy members have a deficiency of C IV absorption. Similarly, we find that the larger groups of galaxies tend to have little or no O VI absorption. In our study, the largest group galaxy environment (with eight members), J022815-405714 at $z_{\text {abs }}=0.2677$, had an upper limit of $W_{r}=0.016 \AA$ and was classed as a O VI non-absorber. Additionally, the second largest group (with five members), J040748 -121136 at $z_{\text {abs }}=0.0919$, was a weak O VI absorber with $W_{r}=0.08 \AA$. Thus, it is possible that the gas traced by $\mathrm{OVI}$ in dense group environments could also be undergoing ram pressure or tidal stripping, leading to the gas being removed from the CGM. Future studies could focus on building a complete sample of groups with a range of member galaxies to investigate this trend.

While the effect of group environments on the CGM was less evident in the covering fractions, we further support the differences in the average equivalent width of the two samples with the kinematics, which were found to be significantly different from isolated environments. The TPCFs showed that, on average, group environment absorption is narrower than isolated galaxy absorption with a difference between the two populations of $10 \sigma$. To quantify this, $\Delta v(90)$ for the group environments $\left(153_{-18}^{+21} \mathrm{~km} \mathrm{~s}^{-1}\right)$ is roughly two-thirds that of the isolated sample $\left(236 \pm 15 \mathrm{~km} \mathrm{~s}^{-1}\right)$. Stocke et al. (2014) similarly found that the total absorption profiles of $\mathrm{O}$ VI absorbers were narrower for group environments. However, the 
individual clouds within the profiles were represented by broader components, which indicates higher virial temperatures. This narrowing of the absorption profiles, along with potentially smaller average equivalent widths and covering fractions, strongly suggests that the warm CGM does not exist as a superposition of halos as found in $\mathrm{Mg}$ II by Bordoloi et al. (2011). This is further supported by Stocke et al. (2014), who found that $\mathrm{O}$ VI absorbers were not associated with the nearest galaxy in the group environment and favored a mixed CGM scenario. If the group environment CGM properties were due to a superposition of halos, we would expect to see similar or larger velocity widths in group environments compared to the isolated galaxies, which we did not observe.

Similarly to Stocke et al. (2014), we suggest that the regions of warm O VI CGM gas would exist at the interface between the higher virial temperature, diffuse medium of the combined group CGM, and the cooler gas associated with individual group galaxies. We suggest that the narrow absorption profiles of $\mathrm{O} \mathrm{VI}$ in group environments is due to the ionization of lower column density gas in the higher velocity wings of the absorbers. Thus, the O VI gas regions are ionized from the outside of the region's mass, effectively forming an "onion skin" effect, with higher ionization states on the outside and decreasing inwards. This would create a shell of higher ionized oxygen surrounding the O VI, leading to the observed smaller velocity spread. Similar ideas have been found by Shull et al. (1998, 2003), Tripp et al. (2001), Aracil et al. (2006), Churchill et al. (2012), Kacprzak et al. (2013), Stocke et al. (2014), Stocke et al. (2017), and Stern et al. (2016). Thus, narrower profiles could be the result of a higher virial temperature, causing regions of $\mathrm{O}$ VI to be ionized from the outside inward.

The destruction of $\mathrm{O}$ VI and its existence at interface regions means that $\mathrm{O} \mathrm{VI}$ is an interesting probe of the processes which occur in group environments. More information of the diffuse medium surrounding the interface could be found from higher ionization states such as O VII (Shull et al. 2003). However, at this redshift, O VII is in the x-ray regime and not detectable with current telescopes. Since O VI accounts for such a small fraction of the total baryons and oxygen budget (Peeples et al. 2014; Oppenheimer et al. 2016) investigating higher ionization states such as $\mathrm{O}$ VII, O VIII, Ne VIII, and Mg X could further the understanding of the total baryon contents of the CGM.

\section{Summary and Conclusions}

We compare the CGM for a total of six group galaxy environments to the CGM of 29 isolated galaxies from Kacprzak et al. (2015) associated with O VI absorption. We also have another 12 groups and 38 isolated galaxies with only an upper limit on O VI absorption. Group environments were classified as having at least two galaxies with a line-of-sight velocity separation of less than $1000 \mathrm{~km} \mathrm{~s}^{-1}$, and the nearest member located within 20-350 kpc of the quasar sightline.

We modeled the absorption profiles for the group sample using VPFIT to obtain the column densities, Doppler (b) parameters, and redshifts, from which we calculated the equivalent width and velocity spreads. We directly compared the equivalent widths and covering fractions of the two samples, and used the pixel-velocity TPCF method from Nielsen et al. $(2015,2016)$ to compare the velocity spreads of the group and isolated galaxy samples. Our findings are as follows.
1. The equivalent width of the absorption in group environments follows the previously established trend of decreasing as the impact parameter increases. Larger samples of group galaxies could determine if an anticorrelation exists similar to that found for isolated galaxies.

2. The average equivalent width of absorption associated with group environments is $0.16 \pm 0.02 \AA$ compared to the larger value for isolated galaxies, which is $0.31 \pm$ $0.03 \AA$. The difference between the two populations is $3 \sigma$, which is significant.

3. The covering fraction is $C_{f}=0.33_{-0.17}^{+0.06}$ for group galaxies and $C_{f}=0.43_{-0.06}^{+0.03}$ for isolated galaxies. The covering fraction of absorption associated with group environments is smaller than that for isolated galaxies, but they are consistent within the errors of the populations with a difference of only $0.8 \sigma$.

4. The velocity spread of the absorption profiles of group environments was found to be significantly narrower than the velocity spread of the absorption profiles of isolated galaxies using the TPCF analysis, and a Chi-squared test found that this result is significant at the $10 \sigma$ level. A comparison of the velocity spread between the group sample $\left(\Delta v(90)=154 \pm 19 \mathrm{~km} \mathrm{~s}^{-1}, \Delta v(50)=66 \pm 8 \mathrm{~km} \mathrm{~s}^{-1}\right)$ and the isolated sample $\left(\Delta v(90)=236 \pm 15 \mathrm{~km} \mathrm{~s}^{-1}\right.$, $\Delta v(50)=100 \pm 6 \mathrm{~km} \mathrm{~s}^{-1}$ ) found that the difference in these values between the two samples was at least $2.3 \sigma$.

The narrow velocity spreads in group environments indicate that $\mathrm{O}$ VI exists at boundaries between the more highly ionized and diffuse CGM and the cooler gas, which is in agreement with Shull et al. (1998, 2003) and Stocke et al. (2014, 2017). We find that is it unlikely that the warm and hot CGM exists as a superposition of the galaxy group member halos, unlike the scenario suggested by Bordoloi et al. (2011) for the cool CGM. This idea is consistent with Oppenheimer et al. (2016), who found that $\mathrm{O}$ VI is strongly dependent on mass as more massive halos have larger virial temperatures, indicating that the CGM in group environments is warm enough to ionize oxygen to $\mathrm{O}$ VII or higher. The lower average equivalent width and slightly smaller covering fraction for group environments also suggests that warm gas is being ionized further in group environments.

The O VI absorption toward group environments is markedly different from that detected toward isolated galaxies, although we would benefit from a larger sample size to better understand some of the differences. A study of the higher ionization states of the CGM in group environments would help us to understand the processes which are occurring in group environments; in particular, an investigation of the narrowing of the O VI absorption profiles and the non-superposition of warm CGM halos surrounding group galaxies. Such a study is not currently feasible, as these features would be found in the $\mathrm{x}$-ray regime. However, investigating other high ionization states, located in the UV, would also help to broaden our understanding of the CGM in group environments.

Support for this research was provided by NASA through grants HST GO-13398 from the Space Telescope Science Institute, which is operated by the Association of Universities for Research in Astronomy, Inc., under NASA contract NAS526555. S.K.P. acknowledges support through the Australian Government Research Training Program Scholarship. G.G.K. 
acknowledges the support of the Australian Research Council through the award of a Future Fellowship (FT140100933). S.M. acknowledges support from European Research Council (ERC), Grant Agreement 278594-GasAroundGalaxies. C.W.C. and J.C. acknowledge the support from award 1517831 from the National Science Foundation.

\section{References}

Aracil, B., Tripp, T. M., Bowen, D. V., et al. 2006, MNRAS, 367, 139 Barnes, J. E. 2004, MNRAS, 350, 798

Barnes, J. E., \& Hernquist, L. 1992, ARA\&A, 30, 705

Beasley, A. J., Gordon, D., Peck, A. B., et al. 2002, ApJS, 141, 13

Beuermann, K., Thomas, H.-C., Reinsch, K., et al. 1999, A\&A, 347, 47

Bordoloi, R., Lilly, S. J., Knobel, C., et al. 2011, ApJ, 743, 10

Bridge, C. R., Carlberg, R. G., \& Sullivan, M. 2010, ApJ, 709, 1067

Burchett, J. N., Tripp, T. M., Bordoloi, R., et al. 2016, ApJ, 832, 124

Burchett, J. N., Tripp, T. M., Werk, J. K., et al. 2013, ApJL, 779, L17

Carswell, R. F., \& Webb, J. K. 2014, VPFIT: Voigt Profile Fitting Program Astrophysics Source Code Library, ascl:1408.015

Cen, R., \& Ostriker, J. P. 1999, ApJ, 514, 1

Chen, H.-W., Helsby, J. E., Gauthier, J.-R., et al. 2010, ApJ, 714, 1521

Chen, H.-W., Lanzetta, K. M., Webb, J. K., \& Barcons, X. 2001, ApJ, 559, 654

Chen, H.-W., \& Mulchaey, J. S. 2009, ApJ, 701, 1219

Churchill, C. W. 1997, PhD thesis, Univ. California

Churchill, C. W., Kacprzak, G. G., Steidel, C. C., et al. 2012, ApJ, 760, 68

Cowie, L. L., \& Songaila, A. 1977, Natur, 266, 501

Dekel, A., \& Birnboim, Y. 2006, MNRAS, 368, 2

Ellingson, E., Green, R. F., \& Yee, H. K. C. 1991, ApJ, 378, 476

Fey, A. L., Ma, C., Arias, E. F., et al. 2004, AJ, 127, 3587

Fujita, Y., \& Nagashima, M. 1999, ApJ, 516, 619

Gunn, J. E., \& Gott, J. R., III 1972, ApJ, 176, 1

Healey, S. E., Romani, R. W., Taylor, G. B., et al. 2007, ApJS, 171, 61

Johnson, S. D., Chen, H.-W., \& Mulchaey, J. S. 2013, MNRAS, 434, 1765

Johnson, S. D., Chen, H.-W., \& Mulchaey, J. S. 2015, MNRAS, 449, 3263

Kacprzak, G. G., Churchill, C. W., Evans, J. L., Murphy, M. T., \& Steidel, C. C. 2011, MNRAS, 416, 3118

Kacprzak, G. G., Churchill, C. W., Steidel, C. C., \& Murphy, M. T. 2008, AJ, 135,922

Kacprzak, G. G., Cooke, J., Churchill, C. W., Ryan-Weber, E. V., \& Nielsen, N. M. 2013, ApJL, 777, L11

Kacprzak, G. G., Muzahid, S., Churchill, C. W., Nielsen, N. M., \& Charlton, J. C. 2015, ApJ, 815, 22

Kacprzak, G. G., van de Voort, F., Glazebrook, K., et al. 2016, ApJL, 826, L11

Keel, W. C., Kennicutt, R. C., Jr., Hummel, E., \& van der Hulst, J. M. 1985, AJ, 90, 708

Kriss, G. A. 2011, COS Instrument Science Report 2011-01(v1), Improved Medium Resolution Line Spread Functions for COS FUV Spectra (Baltimore, MD: STScI)
Lanzetta, K. M., Bowen, D. V., Tytler, D., \& Webb, J. K. 1995, ApJ, 442, 538

Larson, R. B., Tinsley, B. M., \& Caldwell, C. N. 1980, ApJ, 237, 692

Li, J., \& Jin, W. 1996, A\&AS, 120, 201

Lilly, S. J., Carollo, C. M., Pipino, A., Renzini, A., \& Peng, Y. 2013, ApJ, 772,119

Maller, A. H., \& Bullock, J. S. 2004, MNRAS, 355, 694

Mo, H. J., \& Miralda-Escude, J. 1996, ApJ, 469, 589

Moore, B., Katz, N., Lake, G., Dressler, A., \& Oemler, A. 1996, Natur, 379,613

Muzahid, S., Kacprzak, G. G., Charlton, J. C., \& Churchill, C. W. 2016, ApJ, 823,66

Muzahid, S., Kacprzak, G. G., Churchill, C. W., et al. 2015, ApJ, 811, 132

Nielsen, N. M., Churchill, C. W., \& Kacprzak, G. G. 2013a, ApJ, 776, 115

Nielsen, N. M., Churchill, C. W., Kacprzak, G. G., \& Murphy, M. T. 2013b, ApJ, 776, 114

Nielsen, N. M., Churchill, C. W., Kacprzak, G. G., Murphy, M. T., \& Evans, J. L. 2015, ApJ, 812, 83

Nielsen, N. M., Churchill, C. W., Kacprzak, G. G., Murphy, M. T., \& Evans, J. L. 2016, ApJ, 818, 171

Nielsen, N. M., Kacprzak, G. G., Muzahid, S., et al. 2017, ApJ, 834, 148

Nulsen, P. E. J. 1982, MNRAS, 198, 1007

Oppenheimer, B. D., Crain, R. A., Schaye, J., et al. 2016, MNRAS, 460, 2157

Oppenheimer, B. D., \& Davé, R. 2008, MNRAS, 387, 577

Peeples, M. S., Werk, J. K., Tumlinson, J., et al. 2014, ApJ, 786, 54

Poggianti, B. M., Fasano, G., Omizzolo, A., et al. 2016, AJ, 151, 78

Prochaska, J. X., Weiner, B., Chen, H.-W., Mulchaey, J., \& Cooksey, K. 2011, ApJ, 740, 91

Rudie, G. C., Steidel, C. C., Trainor, R. F., et al. 2012, ApJ, 750, 67

Savage, B. D., Kim, T.-S., Wakker, B. P., et al. 2014, ApJS, 212, 8

Shull, J. M., Penton, S. V., Stocke, J. T., et al. 1998, AJ, 116, 2094

Shull, J. M., Tumlinson, J., \& Giroux, M. L. 2003, ApJL, 594, L107

Steidel, C. C., Erb, D. K., Shapley, A. E., et al. 2010, ApJ, 717, 289

Stern, J., Hennawi, J. F., Prochaska, J. X., \& Werk, J. K. 2016, ApJ, 830, 87

Stocke, J., Keeney, B., Danforth, C., et al. 2017, arXiv:1702.08554

Stocke, J. T., Keeney, B. A., Danforth, C. W., et al. 2013, ApJ, 763, 148

Stocke, J. T., Keeney, B. A., Danforth, C. W., et al. 2014, ApJ, 791, 128

Thom, C., Werk, J. K., Tumlinson, J., et al. 2011, ApJ, 736, 1

Tripp, T. M., Giroux, M. L., Stocke, J. T., Tumlinson, J., \& Oegerle, W. R. 2001, ApJ, 563, 724

Tully, R. B., Rizzi, L., Shaya, E. J., et al. 2009, AJ, 138, 323

Tumlinson, J., Thom, C., Werk, J. K., et al. 2011, Sci, 334, 948

Veilleux, S., Cecil, G., \& Bland-Hawthorn, J. 2005, ARA\&A, 43, 769

Werk, J. K., Prochaska, J. X., Thom, C., et al. 2012, ApJS, 198, 3

Werk, J. K., Prochaska, J. X., Thom, C., et al. 2013, ApJS, 204, 17

Werk, J. K., Prochaska, J. X., Tumlinson, J., et al. 2014, ApJ, 792, 8

Wu, X.-B., Hao, G., Jia, Z., Zhang, Y., \& Peng, N. 2012, AJ, 144, 49

Zickgraf, F.-J., Engels, D., Hagen, H.-J., Reimers, D., \& Voges, W. 2003, A\&A, 406, 535 